\title{
Risk of postoperative pulmonary complications in adult surgical patients with metabolic syndrome: a systematic review and meta-analysis protocol
}

\author{
Philip Norris $^{1 *}$ (D), Bianca Viljoen ${ }^{1}$, Nicholas Ralph¹, Jeff Gow ${ }^{2,3}$ and Natalie Silvey ${ }^{4}$
}

\begin{abstract}
Background: Metabolic syndrome (MetS) is defined as an accumulation of risk factors that include chronic hypertension, dyslipidaemia, insulin resistance and obesity and leads to an increased risk for diabetes, cardiovascular disease and stroke. MetS is widespread and estimated to affect up to a quarter of the global population. Patients with MetS who undergo surgery are associated with an increased risk of postoperative complications when compared with patients with a non-MetS profile. An emerging body of literature points to MetS being associated with a greater risk of postoperative pulmonary complications (PPC) in the surgical patient. PPC are associated with increased postoperative morbidity and mortality, Intensive care unit (ICU) admission, length of stay (ICU and hospital), health care costs, resource usage, unplanned re-intubation and prolonged ventilatory time.

Methods/design: We will search for relevant studies in the following electronic bibliographic databases: EMBASE, MEDLINE, Cumulative Index to Nursing and Allied Health Literature (CINAHL) and Scopus as well as scan the reference lists of included studies for potential additional literature. Two authors will independently screen titles and abstracts to identify potentially relevant studies for inclusion based on predefined inclusion and exclusion criteria. The Cochrane Collaboration Review Manager (Review Manager 5) statistical software will be used to conduct this systematic review and meta-analysis and generate forest plots to demonstrate comparison of findings across studies included for metaanalysis. Subgroup and sensitivity analysis will be performed to assess the heterogeneity of included studies. A descriptive synthesis of the statistical data will be provided to summarise the results and findings of the systematic review and meta-analysis.

Discussion: This review will be the first to report and summarise the risk for and incidence of PPC in adult patients with MetS undergoing surgery across a range of surgical specialities. The results have the potential to inform the development of evidenced-based interventions to improve the management of PPC in the surgical patient with MetS. Findings from this systematic review and meta-analysis will inform a subsequent Delphi study on priorities and responses to PPC in patients with MetS. We will also disseminate our results through publication in scientific peerreviewed journals, conference presentations and promotion throughout our network of surgical safety champions in clinical settings.
\end{abstract}

\section{Systematic review registration: PROSPERO CRD42019120279.}

Keywords: Metabolic syndrome, Surgery, Complications, Postoperative pulmonary complications, Systematic review, Meta-analysis, Protocol

\footnotetext{
*Correspondence: philip.norris@usq.edu.au

${ }^{1}$ School of Nursing and Midwifery, Faculty of Health, Engineering and

Sciences, University of Southern Queensland, Toowoomba, Australia

Full list of author information is available at the end of the article
}

(c) The Author(s). 2019 Open Access This article is distributed under the terms of the Creative Commons Attribution 4.0 International License (http://creativecommons.org/licenses/by/4.0/), which permits unrestricted use, distribution, and reproduction in any medium, provided you give appropriate credit to the original author(s) and the source, provide a link to the Creative Commons license, and indicate if changes were made. The Creative Commons Public Domain Dedication waiver (http://creativecommons.org/publicdomain/zero/1.0/) applies to the data made available in this article, unless otherwise stated. 


\section{Background}

Metabolic syndrome (MetS) is a global health problem which leads to an increased risk of cardiovascular disease, stroke, non-insulin dependent diabetes mellitus and premature death [1]. Individuals with MetS typically present with symptoms that include elevated blood pressure, insulin resistance, decreased high-density lipoproteins, elevated triglycerides and obesity, particularly abdominal obesity [2, 3]. Typically, an individual is diagnosed with MetS when at least three out of five of these components are present $[4,5]$. However, these components vary slightly according to the definition of MetS used. While the various definitions share the same inherent risk factors, diagnostic criteria diverge slightly across each definition. Historically, two commonly used definitions are from the International Diabetes Federation (IDF), and the National Cholesterol Education Program Adult Treatment Panel III (NCEP:ATPIII) [6, 7]. To overcome the difficulties associated with multiple definitions of MetS, leading health organisations produced a Joint Interim Statement (JIS) to harmonise diagnostic criteria and components of MetS into a unified, widely accepted, and broadly adopted definition as outline in Table 1 [6].

Unifying and using a single diagnostic criteria for MetS is important as previous global estimates of prevalence range between $10 \%$ and $84 \%$ depending on region, age, ethnicity, gender and race [1]. However, MetS is most commonly estimated to be around 20 to $30 \%$ in adult populations within developed economies $[8,9]$. While an accurate prevalence of MetS in surgical patients is not obtainable from the literature, the prevalence of MetS in patients undergoing cardiac surgery alone is estimated at approximately $46 \%[10,11]$. This estimate is almost double that reported in the general population $[12,13]$. A diagnosis of MetS predicts the development of chronic disease and deterioration of health. MetS increases the risk for all-cause mortality 1.5 fold, stroke twofold, cardiovascular disease threefold, and type two diabetes mellitus

Table 1 Diagnostic criteria for the metabolic syndrome

\begin{tabular}{|c|c|}
\hline Measure & Categorical cut-off points \\
\hline Elevated waist circumference & $\begin{array}{l}\text { Population- and country-specific } \\
\text { definitions }\end{array}$ \\
\hline $\begin{array}{l}\text { Elevated triglycerides (drug } \\
\text { treatment for elevated triglycerides } \\
\text { is an alternate indicator) }\end{array}$ & $\geq 150 \mathrm{mg} / \mathrm{dL}(1.7 \mathrm{mmol} / \mathrm{L})$ \\
\hline $\begin{array}{l}\text { Reduced HDL-C (drug treatment } \\
\text { for reduced } \mathrm{HDL}-\mathrm{C} \text { is an alternate } \\
\text { indicator) }\end{array}$ & $\begin{array}{l}<40 \mathrm{mg} / \mathrm{dL}(1.0 \mathrm{mmol} / \mathrm{L}) \text { in males; } \\
<50 \mathrm{mg} / \mathrm{dL}(1.3 \mathrm{mmol} / \mathrm{L}) \text { in females }\end{array}$ \\
\hline $\begin{array}{l}\text { Elevated blood pressure } \\
\text { (antihypertensive drug treatment } \\
\text { in a patient with a history of } \\
\text { hypertension is an alternative } \\
\text { indicator }\end{array}$ & $\begin{array}{l}\text { Systolic } \geq 130 \text { and/or diastolic } \geq \\
85 \mathrm{mmHg}\end{array}$ \\
\hline $\begin{array}{l}\text { Elevated fasting glucose (drug } \\
\text { treatment of elevated glucose in } \\
\text { an alternate indictor) }\end{array}$ & $\geq 100 \mathrm{mg} / \mathrm{dL}$ \\
\hline
\end{tabular}

fivefold [1, 14-16]. MetS also predicts the development of a range of chronic diseases including non-alcoholic steatohepatitis, neurological disorders and malignant neoplasms [17]. Having MetS is further associated with increased complications in patients undergoing surgery compared to patients with a non-MetS profile. Frequently reported perioperative adverse events indicate that patients with MetS are associated with increased rates of postoperative morbidity compared to patients without it specifically, infectious, cardiovascular and renal postoperative adverse events $[10,18,19]$.

One particular under-researched area of burden predicted by MetS relates to postoperative pulmonary complications (PPC) after surgery. PPC occur commonly and increase patient mortality and morbidity as well as being associated with increased health-care costs. One in five patients who develop a PPC will die within 30 days of major surgery compared to $0.2-3 \%$ without [20] and an observational study has shown that there are long-term significant differences in mortality rates at 1 and 5 years for patients who have had a PPC [20]. Length of hospital stay is also increased with resultant increased morbidity and this also increases healthcare costs significantly [21]. Increasingly, there are reports of a heightened risk of PPC among surgical patients' with MetS. For example, in a study of 158,405 patients undergoing bariatric surgery, the complications of atelectasis, pleural effusions, pneumonia, ARDS and respiratory failure were associated with a significantly higher percentage of occurrences in patients with MetS compared to patients without a MetS diagnosis [22]. For patients undergoing hepatic surgery, having MetS increases the risk of unplanned re-intubation and prolonged ventilatory time twofold [23]. In a study reporting on outcomes for patients undergoing cardiac surgery, rates of PPC and length of ventilatory time were significantly higher in patients with a MetS profile [24]. In another retrospective cohort study of 310,280 patients undergoing non-cardiac surgery, the risk for PPC was approximately 1.5 to threefold higher in patients when accompanied by a MetS diagnosis [25]. MetS was found to be an independent positive predictor of PPC in patients undergoing abdominal surgery [26]. Increased rates of PPC were also reported in patients diagnosed with MetS who underwent lumbar spinal fusion and shoulder arthroplasty [27, 28]. An emerging body of literature appears to associate MetS with a greater risk of PPC in the surgical patient. However, no systematic review has been performed to date meaning the risk of PPC in MetS patients is not well understood. Additionally, there are few studies of interventions specifically targeting the needs of the surgical patient with MetS. Our proposed systematic review and meta-analysis will therefore be the first of its kind to synthesise and critically appraise the current evidence base. We will quantify the risk of PPC in patients with metabolic syndrome versus those without metabolic syndrome in surgical populations. 


\section{Objectives}

The objective of this review is to evaluate the effect of metabolic syndrome on the occurrence of PPC in adult surgical patients versus those without metabolic syndrome.

\section{Methods}

\section{Protocol}

This systematic review will follow the Preferred Reporting Items for Systematic Review and Meta-Analysis (PRISMA) recommendations [29]. The systematic review protocol has been registered in the International Prospective Register of Systematic Reviews (PROSPERO CRD42019120279). A PRISMA-P file is attached (see Additional file 1) [30].

\section{Eligibility criteria}

\section{Criteria for considering studies}

Study inclusion criteria This review will include peerreviewed literature that is published in electronic bibliographic databases. Included studies must examine the relationship between adult surgical patients 18 years of age and older diagnosed with MetS and the risk for and incidence of PPC in this population. Studies will be restricted by design with only retrospective and prospective observational studies, cohort studies and case-control studies being eligible for inclusion to obtain a comprehensive overview of the existing evidence base.

\section{Population}

Surgical patients diagnosed with MetS versus surgical patients without MetS.

\section{Outcomes}

The primary outcome will be the incidence of PPC in adult surgical patients diagnosed with MetS at key surgical/clinical time intervals. We will report outcomes of interest as odds ratio, relative risk or incidence rate ratios. We will only record outcomes reported within the first 30 days after surgery.

PPC will be defined using the European Perioperative Clinical Outcomes (EPCO) definitions [31]. These include respiratory infection, respiratory failure, pleural effusion, atelectasis, pneumothorax, bronchospasm, aspiration pneumonitis, pneumonia, acute respiratory distress syndrome (ARDS), tracheobronchitis, pulmonary oedema, exacerbation of pre-existing lung disease and pulmonary embolism.

We will also record secondary outcomes of interest as follows:

- Unplanned re-intubation within 30 days

- Prolonged ventilatory time $>72 \mathrm{~h}$

\section{Search strategy}

We will conduct a comprehensive literature search of the following electronic databases: MEDLINE (Medical Literature Analysis and Retrieval Online), CINAHL (Cumulative
Index to Nursing and Allied Health Literature), EMBASE (ExerptaMedica Database) and Scopus to identify studies pertinent to the review. A date restriction from January 1, 1998 until present, will be implemented to reflect the first formal definition for metabolic syndrome which was developed in 1998 by the World Health Organization and informed every subsequent definition of MetS since [6].

The types of studies that will be analysed and included are retrospective or prospective observational studies, cross-sectional studies, cohort studies and case control studies. The types of studies that will be excluded are letters, abstracts, conference papers and editorials. The initial search terms reflect how metabolic syndrome is commonly referred to in the literature. These keywords will include the Medical Subject Heading for "metabolic syndrome" and a range of synonyms used to represent surgery and postoperative pulmonary complications. Search terms will be limited to peer-reviewed full text-articles, in English language with no geographical restriction (see Appendix 1).

To ensure that all relevant studies are included, a manual search of citations and references of eligible studies will also be conducted. Resulting references will be exported separately and provided to two reviewers (PN and BV) for independent review. Where necessary, study authors will be contacted for missing information. To ensure impartiality the inclusion and exclusion criteria will be engaged continuously (see Appendix 2).

\section{Selection of studies}

Following the search, studies selected for inclusion will be collated into a citation management program (Endnote X8). Duplicates will be removed and stored separately (Endnote $\mathrm{X} 8$ ). Two reviewers (PN and BV) will independently screen titles and abstracts for assessment against the inclusion criteria and exclude all studies that do not meet these criteria. Any disagreement will be resolved through discussion and consensus. After the initial screening for studies, two reviewers (PN and BV) will retrieve the full text of selected studies and assess against the inclusion criteria. Full-text studies that meet the inclusion criteria will be imported into Endnote X8. Full text studies that do not meet the inclusion criteria will be excluded and reasons for exclusion will be provided in an appendix in the final systematic review report. Included studies will undergo a process of critical appraisal. Any disagreements that arise between the reviewers will be resolved through discussion, or with a third reviewer (NR). The results of the search will be reported in the review and presented in a PRISMA flow diagram, detailing the steps taken in the full systematic review. The authors will be contacted for missing data where required.

\section{Data extraction and management}

Two reviewers (PN and BV) will independently extract data from the included studies into Cochrane Collaboration Review Manager (Review Manager 5) to ensure consistency 
while reducing bias and improving validity and reliability [32]. Any inconsistences between reviewers will be resolved through consensus and a third reviewer will be consulted if agreement cannot be reached. Study authors will be contacted to obtain additional or missing data.

Extracted data will include the following:

1. Study details: title, journal, year, city and country where the research was undertaken.

2. Participant demographics: sample size, group size (e.g. metabolic syndrome group versus nonmetabolic syndrome group), reported complications, metabolic syndrome diagnostic criteria applied, type of surgery, population demographics.

3. Methods: study aim, data collection method, study recruitment, design and sampling methods, study eligibility as dictated by the inclusion criteria

4. Outcome measures: estimates of cumulative incidence and incidence rate of PPC in adult surgical patients diagnosed with MetS.

5. Limitations: study biases as identified by a risk of bias tool for observational studies and limitations as identified by the study authors.

\section{Risk of bias}

The methodological quality and bias of the included studies will be assessed using the Newcastle-Ottawa Quality Assessment Scale (NOS) [33]. The NOS will evaluate nonrandomised studies included in systematic reviews and meta-analyses across three quality parameters: study selection, comparability of the population and a determination of whether the exposure or outcome includes risk of bias [32]. NOS assesses the quality of each study and provides a maximum score of nine points. Studies identified as having a NOS greater than or equal to seven are considered high quality. Studies between five and six points are considered as being of fair or moderate quality. Studies that are assessed as having a NOS score of less than five points represent a high risk of bias [32]. Two investigators (PN and BV) will independently assess the risk of bias for included studies using NOS quality criteria.

\section{Data collection and analysis}

We will conduct data analysis using the Cochrane Collaboration Review Manager (Review Manager 5) statistical software to generate forest plots to demonstrate comparison of findings across studies included for metaanalysis. Meta-analysis will be performed if two or more comparable studies are identified for each outcome of interest. We anticipate clinical and methodological diversity as population and study characteristics will vary across trials. The statistical heterogeneity of studies included for meta-analysis will be assessed by calculating the $P$ value from the chi-squared test for homogeneity and the $I^{2}$ statistic. A $P$ value of $<0.10$ will be used to determine statistical significance [28]. In the presence of substantial heterogeneity $\left(I^{2}\right.$ is $\geq 50 \%$ ), we will pool studyspecific estimates using a random effects model and according to whether variables are dichotomous or continuous [34]. For dichotomous variables, individual study data will be pooled and examined using the MantelHaenszel to examine the overall association between the exposure and outcome [35]. For continuous variables, we will group measures using the inverse variance approach to pool the standardised mean difference where studies have employed different measures to calculate the outcome of interest or weighted mean difference if studies report efficacy in terms of a continuous outcome measurement [36].

If heterogeneity is low $\left(I^{2}\right.$ is $\left.\leq 50 \%\right)$, pooled estimates will be calculated using a fixed-effects model [36]. Measures of relative effect will be expressed as odds ratio (OR) and 95\% confidence intervals (CIs) for dichotomous outcomes while standard mean differences and 95\% CIs will be calculated for continuous outcomes. If statistical heterogeneity is present, subgroup analysis (e.g. population according to the MetS definition categories, surgery type, type of PPC, age and gender) will be undertaken and meta-regression performed to explore effect measure modification where the modifiers are study level covariates. Sensitivity analysis will be performed to identify study level categorical variables that characterise the occurrence of the outcome of interest. We will also assess the impact of outlier studies on the pooled estimates of reported outcome measures among the population of interest by performing outlier analysis.

If there are ten or more studies included in the metaanalysis, the reviewers will assess for potential publication bias using funnel plots and Egger's test [37]. Any discrepancies of quality assessment between the two reviewers (PN and $\mathrm{BV}$ ) will be resolved through discussion and consensus or by a third reviewer (NR).

\section{Discussion}

To our knowledge, this systematic review and metaanalysis will be the first to evaluate the risk of PPC following surgery in patients with and without MetS. If MetS increases the probability of complications following surgery, patients should be made aware of these risks while clinicians should develop interventions to reduce or eliminate the likelihood of PPC occurring. Following our review, we will use numerous strategies to disseminate our findings. Examples include conference presentations, media releases, meetings with healthcare leaders and expansion of our current research program in metabolic syndrome [16] to include PPC intervention development. We will also use findings from our review to inform a subsequent Delphi study as described in [38] to identify expert consensus on priorities for improving outcomes among surgical patients with metabolic syndrome. 


\section{Appendix 1}

Table 2 Example search strategies

\begin{tabular}{|c|c|}
\hline Search Date & MEDLINE search strategy \\
\hline January 06, 2019 & $\begin{array}{l}\text { (("surgical procedures, operative"[MeSH Major Topic] OR "surgery"[Title/Abstract]) AND } \\
\text { ("metabolic syndrome"[MeSH Major Topic] OR "metabolic syndrome"[Title/Abstract])) AND } \\
\text { (((“lung"[MeSH Major Topic] OR "respiratory therapy"[MeSH Major Topic]) OR "lung"[Title/Abstract]) } \\
\text { OR "pulmonary"[Title/Abstract])AND ("1998/01/01"[PDAT]: "2019/01/18"[PDAT]) AND } \\
\text { ("1998/01/01"[PDAT]: "2019/01/18"[PDAT]) }\end{array}$ \\
\hline Search Date & CINAHL search strategy \\
\hline January 06, 2019 & $\begin{array}{l}\text { (((TI Metabolic syndrome) OR (AB metabolic syndrome)) AND (((TI surgical procedures) OR } \\
\text { (AB surgical procedures) OR ((TI operative) OR (AB operative)) OR ((TI surgery) OR (AB surgery) } \\
\text { AND (((TI Metabolic syndrome) OR (AB metabolic syndrome)) AND (((TI respiratory therapy) OR } \\
\text { (AB respiratory therapy)) OR ((TI lungs) OR (AB lungs)) OR ((pulmonary) OR (AB pulmonary))) with } \\
\text { limiters of a published date from 1998/01/01 }\end{array}$ \\
\hline Search Date & ScienceDirect search strategy \\
\hline January 06, 2019 & $\begin{array}{l}\text { title-abs-key (metabolic syndrome) AND title-abs-key (surgical procedures*) OR title-abs-key } \\
\text { (operative*) OR title-abs-key (surgery*) AND title-abs-key (respiratory therapy*) OR title-abs-key } \\
\text { (lung) OR title-abs-key (pulmonary) AND LIMIT-TO (yearnav, "2019, 2018, 2017, 2016, 2015, 2014, } \\
\text { 2013, 2012, 2011, 2010, 2009, 2008, 2007, 2006, 2005, 2004, 2003, 2002, 2001, 2000, 1999, 1998") } \\
\text { AND LIMIT-TO (contenttype, "JL, BS", "Journal"). }\end{array}$ \\
\hline Search Date & Embase Search strategy \\
\hline January 06, 2019 & $\begin{array}{l}\text { 'metabolic syndrome':ab,ti AND surgical procedures: ab,ti OR operative: ab,ti OR surgery ab,ti AND } \\
\text { pulmonary:ab,ti OR respiratory therapy:ab,ti OR lungs ab.ti[1998-2019]/py AND [english]/lim } \\
\text { AND [humans]/lim }\end{array}$ \\
\hline
\end{tabular}

\section{Appendix 2}

Table 3 Example inclusion and exclusion criteria

\begin{tabular}{ll}
\hline Example inclusion criteria & Example exclusion criteria \\
\hline Study population & Study population \\
- Adult patients (18years or older) & - Surgical patients $<18$ years of age \\
- Adult patients with a diagnosis of metabolic syndrome as per author definition & - Animals \\
- Adult patients undergoing moderate or highly invasive surgery of all types & - Pregnant women \\
Study surgical procedure & Study surgical procedure \\
- Adult patients undergoing moderate or highly invasive surgery of all types & - Minor procedures \\
Study design & - Caesarean section \\
- Observational studies (e.g. cohort studies, case-control studies) & - Trauma surgery \\
- Published peer-reviewed articles in the English language & - Day surgical procedures \\
& - Cataract procedures \\
& - Plastic surgery \\
& - Cosmetic surgery \\
& - Cardiac catheterisation \\
& - Cystoscopy procedures \\
& - Endoscopy procedures \\
& - Colonoscopy procedures \\
- Lesion removal \\
Study design \\
- Interventional studies
\end{tabular}




\section{Supplementary information}

Supplementary information accompanies this paper at https://doi.org/10. 1186/s13643-019-1241-z.

\section{Additional file 1. PRISMA-P Checklist.}

\section{Abbreviations}

ARDS: Acute respiratory distress syndrome; CABG: Coronary artery bypass graft; CINAHL: Cumulative Index to Nursing and Allied Health Literature; Cls: Confidence intervals; EPCO: European Perioperative Clinical Outcomes; HDL: High-density lipoprotein; ICU: Intensive care unit; IDF: International Diabetes Federation; JIS: Joint Interim Statement; MetS: Metabolic syndrome; NCEP:ATPIII: National Cholesterol Education Program Panel III; NOS: Newcastle Ottawa Scale; OR: Odds ratio; PPC: Postoperative pulmonary complications; PRISMA-P: Preferred Reporting Items for Systematic Review and Meta-Analysis Protocols

\section{Acknowledgments}

Not applicable.

\section{Authors' contributions}

PN developed the premise of the paper and the study methodology based on earlier work published by PN and NR. PN and BV drafted the protocol according to the agreed structure. NR, JG and NS drafted, reviewed and edited the protocol. PN and BV will screen identified literature, conduct data extraction and analysis of the finding of the review. All authors read and approved the final manuscript.

\section{Authors' information}

Philip Norris is an academic and PhD candidate at the University of Southern Queensland. He is an experienced intensive care clinician and has a keen interest in clinical research, metabolic syndrome and improving surgical outcomes. Bianca Viljoen is an academic and PhD candidate at the University of Southern Queensland. She has experience in a variety of health care settings and has an active interest in clinical research, patient outcome management and professional development. Professor Jeff Gow is a health economist and member of the project team. A/Prof Ralph is the Senior Manager for Health Systems and Psycho-Oncology at the Cancer Council QLD. Dr. Silvey is an Anaesthetic Registrar at Imperial College London.

\section{Funding}

Not applicable.

\section{Availability of data and materials}

Not applicable as no datasets have been compiled as of yet.

\section{Ethics approval and consent to participate}

Not applicable.

\section{Consent for publication}

Not applicable.

\section{Competing interests}

The authors declare that they have no competing interests.

\section{Author details}

${ }^{1}$ School of Nursing and Midwifery, Faculty of Health, Engineering and Sciences, University of Southern Queensland, Toowoomba, Australia. ${ }^{2}$ School of Commerce, Faculty of Business, Education, Law and Arts, University of Southern Queensland, Toowoomba, Australia. ${ }^{3}$ School of Accounting, Economics and Finance, Durban, South Africa. ${ }^{4}$ Imperial School of Anaesthesia, London, UK.

Received: 3 April 2019 Accepted: 20 November 2019 Published online: 06 December 2019

\section{References}

1. O'Neill S, O'Driscoll L. Metabolic syndrome: a closer look at the growing epidemic and its associated pathologies. Obes Rev. 2015;16(1):1-12.
2. Srikanthan K, Feyh A, Visweshwar H, Shapiro Jl, Sodhi K. Systematic review of metabolic syndrome biomarkers: a panel for early detection, management, and risk stratification in the west Virginian population. Int J Med Sci. 2016;13(1):25-38.

3. Roberts CK, Hevener AL, Barnard RJ. Metabolic syndrome and insulin resistance: underlying causes and modification by exercise training. Compr Physiol. 2013;3(1):1-58

4. Alberti KG, Eckel RH, Grundy SM, Zimmet PZ, Cleeman II, Donato KA, et al. Harmonizing the metabolic syndrome: a joint interim statement of the international diabetes federation task force on epidemiology and prevention; National Heart, Lung, and Blood Institute; American Heart Association; world heart federation; international atherosclerosis society; and International Association for the Study of obesity. Circulation. 2009;120(16): 1640-1645.

5. Huang PL. A comprehensive definition for metabolic syndrome. Dis Model Mech. 2009;2(5-6):231-7.

6. Kassi E, Pervanidou P, Kaltsas G, Chrousos G. Metabolic syndrome: definitions and controversies. BMC Med. 2011;9:48.

7. Zimmet P, Alberti G, Kaufman F, Tajima N, Silink M, Arslanian S, et al. The metabolic syndrome in children and adolescents. Lancet (London). 2007; 369(9579):2059-61.

8. Grundy SM. Metabolic syndrome pandemic. Arterioscler Thromb Vasc Biol. 2008;28(4):629-36

9. Moreira GC, Cipullo JP, Ciorlia LAS, Cesarino CB, Vilela-Martin JF. Prevalence of metabolic syndrome: association with risk factors and cardiovascular complications in an urban population. PloS one. 2014;9(9):e105056-e.

10. Echahidi N, Pibarot P, Despres JP, Daigle JM, Mohty D, Voisine P, et al. Metabolic syndrome increases operative mortality in patients undergoing coronary artery bypass grafting surgery. J Am Coll Cardiol. 2007:50(9):843-51.

11. Kajimoto K, Kasai T, Miyauchi K, Hirose H, Yanagisawa N, Yamamoto T, et al. Metabolic syndrome predicts 10-year mortality in non-diabetic patients following coronary artery bypass surgery. Circ J. 2008;72(9):1481-6.

12. Guize L, Thomas F, Pannier B, Bean K, Jego B, Benetos A. All-cause mortality associated with specific combinations of the metabolic syndrome according to recent definitions. Diabetes Care. 2007;30(9):2381.

13. Moebus S, Balijepalli C, Lösch C, Göres L, von Stritzky B, Bramlage P, et al. Age- and sex-specific prevalence and ten-year risk for cardiovascular disease of all 16 risk factor combinations of the metabolic syndrome - a crosssectional study. Cardiovasc Diabetol. 2010;9:34.

14. Mottillo S, Filion KB, Genest J, Joseph L, Pilote L, Poirier P, et al. The metabolic syndrome and cardiovascular risk a systematic review and metaanalysis. J Am Coll Cardiol. 2010;56(14):1113-32.

15. Khosravi A, Sadeghi M, Barghikar M. Which components of metabolic syndrome have a greater effect on mortality, CVA and myocardial infarction, hyperglycemia, high blood pressure or both? Adv Biomed Res. 2017;6:121.

16. Norris P, Ralph N, Moloney C. Does metabolic syndrome predict surgical complications? A protocol for a systematic review and meta-analysis. Syst Rev. 2017:6(1):115.

17. Kaur J. A comprehensive review on metabolic syndrome. Cardiol Res Pract. 2014;2014:943162

18. Tzimas P, Petrou A, Laou E, Milionis H, Mikhailidis DP, Papadopoulos G. Impact of metabolic syndrome in surgical patients: should we bother? Br J Anaesth. 2015;115(2):194-202.

19. Hong S, Youn YN, Yoo KJ. Metabolic syndrome as a risk factor for postoperative kidney injury after off-pump coronary artery bypass surgery. Circ J. 2010;74(6):1121-6.

20. Ramachandran SK, M.D., F.R.C.A., Nafiu OO, M.D., Ghaferi A, M.D., Tremper KK M.D., Ph.D., Shanks A, M.S., Kheterpal S, M.D., M.B.A. Independent predictors and outcomes of unanticipated early postoperative tracheal intubation after nonemergent, noncardiac surgery. Anesthesiology 2011;115(1):44-53.

21. Fleisher LA, Linde-Zwirble WT. Incidence, outcome, and attributable resource use associated with pulmonary and cardiac complications after major small and large bowel procedures. Perioperative Med. 2014;3(1):7.

22. Schumann R, Shikora SA, Sigl JC, Kelley SD. Association of metabolic syndrome and surgical factors with pulmonary adverse events, and longitudinal mortality in bariatric surgery. Br J Anaesth. 2015;114(1):83-90.

23. Bhayani $\mathrm{NH}$, Hyder $\mathrm{O}$, Frederick W, Schulick RD, Wolgang $\mathrm{CL}$, Hirose $\mathrm{K}$, et al. Effect of metabolic syndrome on perioperative outcomes after liver surgery: a National Surgical Quality Improvement Program (NSQIP) analysis. Surgery. 2012;152(2):218-26. 
24. Özkan S, Özdemir F, Uğur O, Demirtunç R, Balci AY, Kizilay M, et al. The effects of the metabolic syndrome on coronary artery bypass grafting surgery. Cardiovasc J Afr. 2017;28(1):48-53.

25. Glance LG, Wissler R, Mukamel DB, Li Y, Diachun CA, Salloum R, et al. Perioperative outcomes among patients with the modified metabolic syndrome who are undergoing noncardiac surgery. Anesthesiology. 2010; 113(4):859-72

26. Macić-Džanković A, Mujaković A, Čelik D, Vukotić E, Džanković F, Hamzić S, et al. Metabolic syndrome as independent predictor of respiratory complications after abdominal surgery. Folia Med Facultatis Med Univ Saraeviensis. 2014:49(2):116-121.

27. Memtsoudis SG, Kirksey M, Ma Y, Chiu YL, Mazumdar M, Pumberger M, et al. Metabolic syndrome and lumbar spine fusion surgery: epidemiology and perioperative outcomes. Spine. 2012;37(11):989-95.

28. Murphy AB, Menendez ME, Watson SL, Ponce BA. Metabolic syndrome and shoulder arthroplasty: epidemiology and peri-operative outcomes. Int Orthop. 2016:40(9):1927-33.

29. Moher D, Liberati A, Tetzlaff J, Altman DG, The PG. Preferred reporting items for systematic reviews and meta-analyses: The PRISMA statement. PLoS Med. 2009;6(7):e1000097.

30. Moher D, Shamseer L, Clarke M, Ghersi D, Liberati A, Petticrew M, et al. Preferred reporting items for systematic review and meta-analysis protocols (PRISMA-P) 2015 statement. Syst Rev. 2015;4(1):1.

31. Jammer I, Wickboldt N, Sander M, Smith A, Schultz MJ, Pelosi P, et al. Standards for definitions and use of outcome measures for clinical effectiveness research in perioperative medicine: European perioperative clinical outcome (EPCO) definitions: a statement from the ESA-ESICM joint taskforce on perioperative outcome measures. Eur J Anaesthesiol. 2015; 32(2):88-105.

32. Higgins JPT, Thomas J, Chandler J, Cumpston M, Li T, Page MJ, Welch VA (editors). Cochrane Handbook for Systematic Reviews of Interventions version 6.0 (updated July 2019). Cochrane, 2019. Available from www. training.cochrane.org/handbook.

33. Wells GS, B; O'Connell, D; Pterson, J; Welch, V; Losos, M; Tugwell, P. The Newcastle-Ottawa Scale (NOS) for assessing the quality of nonrandomised studies in meta-analyses 2018. Available from: http://www.ohri.ca/programs/ clinical_epidemiology/oxford.asp. Accessed 20 Feb 2019.

34. Schroll JB, Moustgaard R, Gøtzsche PC. Dealing with substantial heterogeneity in Cochrane reviews. Cross-sectional study. BMC Med Res Method. 2011;11:22.

35. Borenstein M, Hedges LV, Higgins JPT, Rothstein HR. A basic introduction to fixed-effect and random-effects models for meta-analysis. Res Synth Methods. 2010;1(2):97-111.

36. Riley RD, Higgins JPT, Deeks JJ. Interpretation of random effects metaanalyses. BMJ. 2011;342:d549.

37. Egger M, Smith GD, Schneider M, Minder C. Bias in meta-analysis detected by a simple, graphical test. BMJ. 1997;315(7109):629-34.

38. McMillan SS, King M, Tully MP. How to use the nominal group and Delphi techniques. Int J Clin Pharm. 2016;38(3):655-62.

\section{Publisher's Note}

Springer Nature remains neutral with regard to jurisdictional claims in published maps and institutional affiliations.

Ready to submit your research? Choose BMC and benefit from:

- fast, convenient online submission

- thorough peer review by experienced researchers in your field

- rapid publication on acceptance

- support for research data, including large and complex data types

- gold Open Access which fosters wider collaboration and increased citations

- maximum visibility for your research: over $100 \mathrm{M}$ website views per year

At BMC, research is always in progress.

Learn more biomedcentral.com/submissions 